\title{
Efectos de los recursos tecnológicos en el aprendizaje de las matemáticas
}

| Effects of technological resources on mathematics learning |

\author{
Luis Manuel Barrios Soto \\ Imbs19@hotmail.com \\ IED Calixto Álvarez \\ Barranquilla, Colombia
}

\author{
Mercedes Delgado González \\ merdelgon@gmail.com \\ Universidad de Zulia \\ Maracaibo, Venezuela
}

Recibido: 11 octubre 2020

Aceptado: 22 Abril 2021

\begin{abstract}
Resumen: Las funciones de las Tecnologías de Información y Comunicación (TICs) actualmente en educación, giran en torno a un mejor aprendizaje. El presente artículo tuvo como objetivo estimar los efectos que producen los recursos tecnológicos en el aprendizaje de las matemáticas en estudiantes de $7^{\circ}$ grado de Educación Básica, específicamente en el tema de los polígonos en el plano cartesiano. La investigación siguió una metodología descriptiva-correlacional, con diseño cuasi-experimental de dos grupos y aplicación de pre-prueba y post-prueba. Entre los resultados se destaca que el incremento de la calificación entre la pre y post-prueba, para el grupo control fue $4.99 \%$, mientras que para el experimental fue de $10.36 \%$. Con lo anterior, se logró observar que las tecnologías ayudan a mejorar los procesos de aprendizaje de las matemáticas y tienen un efecto satisfactorio si se usan de forman adecuada.
\end{abstract}

Palabras Clave: Recursos tecnológicos, Tecnologías de Información y Comunicación, aprendizaje, aprendizaje de matemáticas, educación matemática.

Abstract: The functions of Information and Communication Technologies (ICTs) in education currently revolve around better learning. The purpose of this article was to estimate the effects of technological resources on the learning of mathematics in 7th grade elementary school students, specifically on the topic of polygons in the Cartesian plane. The research followed a descriptive-correlational methodology, with a quasi-experimental design of two groups and application of pre-test and posttest. Among the results, it is highlighted that the increase in the score between the pre- and post-test for the control group was $4.99 \%$, while for the experimental group it was $10.36 \%$. With the above, it was possible to observe that technologies help to improve the mathematics learning processes and have a satisfactory effect if they are used appropriately.

Keywords: Technological resources, Information and Communication Technologies, learning, mathematics learning, mathematics education. 


\section{Introducción}

La educación hoy en día está siendo impactada por las tecnologías, las cuales giran torno a un aprendizaje más integral, dependiendo de los objetivos que el docente tenga ante una temática especifica o en crear un vínculo entre lo teórico y práctico. Aparicio (2018) plantea que "la incorporación de las Tecnologías de la Información y la Comunicación a la Sociedad del Conocimiento ha generado una transformación en los escenarios educativos tradicionales, mientras aparecen otros nuevos, la mayoría de ellos con el interés del trabajo colaborativo." (p.76). Con lo anterior, se considera que la inmersión de las TICs en aula de clases es indispensable para mejorar el desarrollo intelectual de los educandos, estableciendo una relación entre los procedimientos manuales y virtuales, cambiando con ello algunas prácticas pedagógicas que tienden a poner al docente como centro y fuente del conocimiento.

El uso de recursos tecnológicos debe ser un componente del currículo escolar, el cual ayuda a desarrollar temáticas de alta complejidad y elaborar nuevas conjeturas que potencialicen el desarrollo del pensamiento matemático en los estudiantes, incluyendo los nuevos retos sociales y mundiales que permiten a los alumnos ser partícipes de acontecimientos actuales. El presente artículo de investigación es producto de un conjunto de intereses obtenidos en el desarrollo de un proyecto de investigación dentro de la Maestría en Matemática, Mención Docencia perteneciente a la División de Estudios para Graduados de la Facultad de Humanidades y Educación de la Universidad del Zulia.

El propósito del artículo es presentar los resultados obtenidos al implementar las Tecnologías de la Información y la Comunicación en el aula de clases, evidenciando el aprendizaje significativo en los estudiantes al momento de utilizar dichas tecnologías como herramienta imprescindible dentro de sus procesos de aprendizaje. Además, el proyecto de investigación tuvo como objetivo estimar los efectos que producen los recursos tecnológicos en el aprendizaje de las matemáticas, específicamente en el tema de los polígonos en el plano cartesiano. Se llevó a cabo con estudiantes de séptimo año de la Educación Básica de una institución educativa colombiana. Todos los participantes tenían edades de 12 a 13 años.

\section{Descripción del problema}

La educación mundial busca formar hombres integrales en las diversas dimensiones humanas. Hombres que construyan y sean parte de una comunidad con progresos culturales, políticos, económicos, ambientales, tecnológicos y otros; permitiendo que el individuo crezca en un entorno donde interactúe y pueda contribuir al mejoramiento de la escuela y la sociedad. Es por ello que García (2019), plantea que "la educación se nos presenta con muchas incertidumbres y ambigüedades que, eso sí, podremos afrontar con nuevas herramientas tecnológicas que habrían de integrarse en los procesos educativos con el fin de acometer profundos cambios tanto metodológicos como organizativos en nuestros centros educativos" (p.14).

En este sentido, la educación mundial se considera un medio para mejorar el estilo de vida del ser humano, tanto de manera individual como social. Ahora, si nos enfocamos en los países Latinoamericanos, los fines educativos apuntan a los progresos de una sociedad que se debe preparar académicamente y que, a la vez, impulse el avance tecnológico y favorezca el ámbito político y económico. Vázquez (2020) establece que: "la población, socialmente requiere una educación que le permita reflexionar sobre su actuar en este mundo, las necesidades y desafíos a los que se enfrenta continuamente, de esta manera desarrollar la capacidad para generar nuevas formas de colaboración" (p.18). Por lo anterior, la educación debe formar a un hombre de conciencia, sólido en valores y que promueva ideas innovadoras que permitan a su vez, salir del subdesarrollo.

Colombia, al ser parte de los países Latinoamericanos que se preocupan por dar a sus habitantes una educación de calidad, aún sigue siendo parte de las naciones con bajo desarrollo. Sin embargo, el 
Ministerio de Educación Nacional (1994), en la Ley General de la Educación (Ley 115), define que "la Educación es un proceso de formación permanente, personal, cultural y social que se fundamenta en una concepción integral de la persona humana, de su dignidad, de sus derechos y sus deberes" (p.19). Es decir, los fines de la educación colombiana se trazan en la formación de un individuo capaz de intervenir en los diferentes acontecimientos que afecten al país y permitir con esto, una mejor calidad de vida.

Abarcando lo anterior, en Colombia, el estudio de las matemáticas es de suma relevancia para la formación integral de sus habitantes, esto mantiene relación con lo establecido por el Ministerio de Educación Nacional (2002) en cuanto a los Estándares Básicos de Competencias en Lenguaje, Matemáticas, Ciencias y Ciudadanas, apuntando a un ser matemáticamente competente, lo cual está íntimamente ligado con los fines de la educación. En esto, los docentes deben reflexionar, explorar y apropiarse de aspectos como: impartir una matemática rica en la resolución de problemas en el contexto para brindar técnicas, reglas y justificaciones socialmente destacadas $y$, presentar una disciplina organizada y vinculada a los perfiles profesionales, orientando a los estudiantes desde las escuelas en las actividades que exigen las comunidades profesionales del país.

Por lo anterior, las estrategias de enseñanza son importantes para mejorar las competencias de los estudiantes y para Gutiérrez (2018), "una estrategia nos orienta al objetivo, nos da una secuencia racional que permite economizar tiempo, recursos y esfuerzo y, lo más importante, nos da la seguridad de lograr lo que queremos obtener y de la manera más adecuada para ello" (p.85). Por esto, es necesario aclarar que no todas las formas de enseñar son válidas para impartir contenidos específicos. En el caso de las Matemáticas, por ser una disciplina aplicable en muchos campos, el empleo de las estrategias debe estar enfocado en el interés por aplicar lo aprendido a experiencias de vida, donde el alumno logre crear un vínculo entre lo teórico y lo práctico.

Ahora bien, como la educación tiene el objetivo de mejorar el rendimiento de los alumnos, usando herramientas informáticas en el aula de clases si es necesario, cabe en este momento aclarar que el uso de las TICs debe tener un impacto positivo, que permita un estado de transformación y constante cambio en lo relacionado con las estrategias de enseñanza desde la perspectiva didáctica. Es esta la razón por la cual se plantea la siguiente interrogante: ¿Qué efecto producen los recursos tecnológicos en el aprendizaje de las matemáticas?

\section{La Educación y las TICs}

A través del tiempo, las civilizaciones han avanzado en material tecnológico, lo cual, en la actualidad, resulta ser un componente protagónico dentro de la construcción del conocimiento, pero que también, ha permitido mejorar los aspectos relacionados con la enseñanza y el aprendizaje en las escuelas y universidades de todo el mundo, haciendo frente a los diversos contextos humanos por medio de la innovación. Aparicio (2018) exponen que:

Comprender el potencial pedagógico de las TIC e integrarlas en las estrategias de enseñanza se ha convertido en el gran reto de la educación actual. Su concepción artefactual y mediadora de las actividades educativas exige que sean consideradas en cuanto su orientación hacia fines donde su presencia adquiera significado. (p.76)

Más reciente, se tiene que Aguiar, Velázquez, y Aguiar (2019) opinan que "las TIC tienen un rol ineludible en la práctica docente del siglo XXI. Los estudiantes manejan mucha tecnología; aprenden mejor con herramientas tecnológicas. Es necesaria una dirección de innovación, porque se considera un ámbito trascendente del quehacer educativo" (p.18). 
Con lo anterior, se ha llegado a entender que las instituciones educativas que vinculan a su currículo el uso de las tecnologías de la información y la comunicación como herramienta de aprendizaje, logran proponer actividades relacionadas con el contexto real, cotidiano y natural a sus estudiantes, contribuyendo a mejorar las competencias intelectuales a través de un aprendizaje significativo que permite el enfrentamiento a diferentes retos dentro de la educación misma. En este sentido, las tecnologías de la información y comunicación, están modificando los paradigmas educativos convencionales y generando mejores herramientas dentro de los modelos de enseñanza, envolviendo a su vez, las dimensiones multiculturales y derrocando también, las barreras de tiempo y espacio.

Por otra parte, los recursos tecnológicos han mejorado los procesos de aprendizaje de los estudiantes, de tal manera, que se ha superado la educación tradicional o memorística. Hoy en día se desea educandos críticos socialmente, que intervengan en los procesos culturales y que éstos mismos le encuentren sentido a la apropiación del conocimiento que se le imparte.

En este orden ideas, las tecnologías de la información y la comunicación despiertan un mecanismo interactivo que motiva el uso de la imaginación y la curiosidad en el alumno, estas últimas son facultades humanas que permiten transformar e incluso profundizar las ideas dentro del conocimiento. Además, existen variadas formas de manejar estas tecnologías en el aula, entre ellas tenemos la internet, el hardware, el software, los blogs, portafolios y pizarra electrónica.

Si se abordan las herramientas mencionadas anteriormente, se obtiene, según investigaciones, que la percepción de los estudiantes acerca del uso de recursos tecnológicos, es que son interesantes, divertidos, fáciles y concretos (Hermann, Apolo y Molano, 2019).

Otras herramientas utilizadas con fines educativas son los hardware y software, entendiendo que el primero facilita manejar la información de manera estratégica, aquí se encuentran las cámaras digitales, escáner, dispositivos de audio y video. A su vez, los softwares educativos convierten el computador en una herramienta útil para el proceso denominado enseñanza y aprendizaje, ya que los mismos estudiantes pueden interactuar con los conocimientos que el docente estipule necesarios en el desarrollo de las temáticas abarcadas en el aula, "el software educativo es más que una tarea de ingenieros, ya que, es necesario extrapolar al ámbito digital lo que el docente realiza diariamente, tomando en consideración, la producción de los aspectos pedagógicos, informáticos y comunicativos" (Fernández, Riveros y Montiel, 2017, p.18).

Por otra parte, a medida que avanza el tiempo son creados millones de páginas web, blogs o weblogs, aulas virtuales y softwares educativos. Tantos, que su crecimiento exponencial se debe de cierta manera a todas las herramientas informáticas diseñadas para la web. Sin embargo, según Suárez (2018), este conjunto importante de recursos, pueden ser utilizados para la enseñanza y aprendizaje de la Matemática, los cuales dependen de una variedad de factores; la introducción de novedosos ambientes virtuales de aprendizaje, sustentados en innovadores abordajes metodológicos que permitan un verdadero aprovechamiento de todas las potenciales de las TIC en los procesos educativos.

Hoy en día, muchas escuelas están actualizando sus aulas al introducir algunas de las herramientas mencionadas hasta el momento, pero igualmente están recurriendo a un instrumento mucho más sofisticado que puede mezclar todas ellas, esta herramienta es la pizarra electrónica, también conocida como tablero digital o Pizarra Digital Interactiva (PDI). Cala et al., (2018), exponen que el usar las pizarras electrónicas tiene como ventajas:

1. El uso de las PDI ayuda al alumno a entender mejor el contenido de los temas;

2. Su uso de la PDI aumenta la participación del alumno en clases;

3. El empleo de la PDI hace las clases más interesantes e interactivas;

4. Aumenta la atención del alumno;

5. El profesor dispone de herramientas que facilitan el proceso de enseñanza; 
6. La PDI aumenta las oportunidades de participación y discusión en las clases;

7. Se trata de una tecnología sencilla de utilizar;

8. Para el docente es más laborioso planificar una clase con la PDI;

9. El uso de la pantalla interactiva facilita una renovación metodológica que promueve la innovación didáctica. (p.65)

Por último, este instrumento tecnológico es visto como parte del futuro en todas las escuelas, ya que facilita un vínculo entre alumno y maestro, permite mejorar el contenido de la temática al usar imágenes, videos, sonidos y otros. Por lo tanto, el tablero digital o pizarra electrónica, da al docente y al alumno los recursos necesarios para la construcción de proyectos, la transmisión de información y la creación de nuevas presentaciones digitales.

\subsection{Recursos tecnológicos}

Un recurso tecnológico, es concebido como el medio que utiliza la tecnología con el fin de obtener un objetivo, estos recursos pueden ser físicos como la computados, la impresora, entre otros, y no físicos como el sistema operativo, el software o aplicaciones. Además, "la incorporación de los recursos tecnológicos en la educación tiene como función ser un medio de comunicación, canal de comunicación e intercambio de conocimiento y experiencias" (Jama y Cornejo, 2016, p.205).

En atención a lo anterior, para la presente investigación se usaron recursos tecnológicos (físicos y no físicos); es decir, se utilizaron computadoras con acceso a internet y con software educativos tales como: GeoGebra y Symbolab; la internet se usó como medio para explorar blogs, páginas web con contenido temático sobre los polígonos en el plano cartesiano, video tutoriales, entre otros.

\subsection{Aprendizaje de las matemáticas mediante TICs}

Se ha demostrado el poder que tiene la tecnología en la enseñanza y aprendizaje de cualquier ciencia. Los beneficios ofrecidos por las tecnologías de la información y la comunicación son bastos, promoviendo el desarrollo de las habilidades básicas y la incorporación de las temáticas a la realidad virtual, siendo esto último, una herramienta poderosa al momento de estudiar situaciones específicas o generales donde no intervengan factores ajenos al fenómeno que se estudia. Así, Rodríguez, Romero y Vergara (2017), mencionan algunos beneficios de implementar las tecnologías de la información y la comunicación en la educación:

Permite la comunicación e interacción en la sociedad. Fomenta la calidad del aprendizaje y del desarrollo de destrezas de la sociedad. Aumenta la productividad económica en la sociedad. Permite el desarrollo y la enseñanza en la educación. Fomentan las habilidades de estudiantes, y científicos. Fomenta la actividad comercial, así como la científica. Permite el desarrollo de nuevos modelos pedagógicos basados en el uso de las capacidades y potencialidades que ofrece la tecnología. Permite la investigación sobre las mismas tecnologías. Propone mejorar u optimizar nuestro control del mundo. (p.5)

Analizando lo expuesto anteriormente, es fácil darse cuenta de que las tecnologías de la información y comunicación no son más que herramientas para el aprendizaje, estas nunca van sustituir al docente. Sin embargo, muchas de las tecnologías pueden ser llevadas al aula para facilitar el trabajo de consulta o poder generar procesos matemáticos de formas instantáneas. Hoy en día, las operaciones rutinarias han pasado a un segundo plano, y es ahí donde las tecnologías de la información y la comunicación, como instrumento facilitador del conocimiento, entra en juego. Además, es importante tener presente 
que muchas de las situaciones problemáticas tratadas en el salón de clases deben despertar en los estudiantes momentos de análisis, interpretación, reflexión, argumentación y a su vez, espacios donde la tecnología intervenga para comprobar los resultados obtenidos.

Ahora bien, existen muchos softwares que permiten obtener información matemática de manera sencilla y eficaz; estos programas facilitan generar procesos arduos en tan solo un segundo, al igual que obtener gráficas en tiempo real. Los usos de estos softwares han forjado mejoras en el aprendizaje de los estudiantes al momento de comprender el comportamiento de ciertas funciones matemáticas o entender procedimientos complejos. Asimismo, cabe resaltar que el uso de software en el área de las matemáticas tiene un sentido mucho mayor del que se cree, por ello Jiménez y Jiménez (2017) expone que:

El profesor tiene que elaborar estrategias que despierten el interés por aprender matemáticas, y la tecnología puede ser la herramienta que permita que los jóvenes construyan su propio conocimiento, en la actualidad el maestro debe buscar una herramienta sencilla de implementar pero que al mismo tiempo desarrolle el pensamiento matemático. (p.13)

Se debe tener en cuenta, con lo expuesto hasta el momento, que las herramientas informáticas son un apoyo fundamental en el proceso de enseñanza y aprendizaje de las matemáticas, las cuales, si son bien utilizadas, mejorarán de manera notable la adquisición del conocimiento matemático por parte de los alumnos, despertando con ello, el interés y la motivación. No obstante, el profesor de la asignatura debe conocer las normas y criterios para escoger la herramienta tecnológica adecuada, dado que, gracias a esto, depende la potencialización de los saberes matemáticos y generar aprendizaje significativo, ya que para González, Abad y Belmonte (2020), "la presencia de las TIC en las aulas ha supuesto que los estudiantes dispongan de una serie de recursos tecnológicos, que le aportan estímulos y permiten motivar su curiosidad para acceder al aprendizaje y a la información de un modo lúdico, visual y creativo" (p.106).

\section{Descripción de los métodos de investigación}

Se tiene como fundamento estudiar los efectos que las Tecnologías de la Información y la Comunicación tienden a manifestar en el Aprendizaje de las Matemáticas, por este motivo se desea tomar un tipo de investigación descriptivo-correlacional, cuyo propósito es detallar claramente los eventos que se lleven a cabo en la escena, sin manipular los resultados o datos provenientes del instrumento utilizado y relacionar dos o más variables midiendo con ello el grado de dependencia. Además, su diseño investigativo de define como cuasi-experimental donde los sujetos inmersos en el estudio cumplen la condición mencionada por Hernández y Mendoza (2018) donde sustentan que las investigaciones con diseño cuasi-experimental no toman grupos al azar, sino que los grupos considerados están bajo las mismas condiciones y se han formado anteriormente, es decir, que los grupos están intactos al inicio del experimento.

Por otra parte, uno de los instrumentos utilizados en la investigación, realizado por los investigadores y llamado "cuestionario sobre tecnologías de información y comunicación", está compuesto por 48 ítem divididos en tres indicadores: I) manejo de las tecnologías de la información y la comunicación con 18 ítems; II) aplicabilidad de las TIC con 12 ítems, y III) aprendizaje de las matemáticas basado en las tecnologías de la información y la comunicación con 18 ítems, permitiéndole anotar a los sujetos de la investigación su apreciación en la parte derecha, categorizada por: siempre, casi siempre, casi nunca y nunca.

Así mismo, la investigación también aplica como instrumentos de recolección de información una preprueba y una post- prueba, también denominadas como pretest y postest. El diseño de estas pruebas, 
tuvo como objetivo comparar las muestras antes y después realizando un tratamiento de variables. La pre-prueba evalúa los conceptos básicos a través de preguntas con múltiples opciones de respuestas y también preguntas abiertas, mientras, la post-prueba evalúa los conceptos adquiridos a través de preguntas de desarrollo, este último test se implementó después de haber utilizado algunas estrategias educativas teniendo en cuenta el uso de las tecnologías de la información y la comunicación (ver Figura 1).

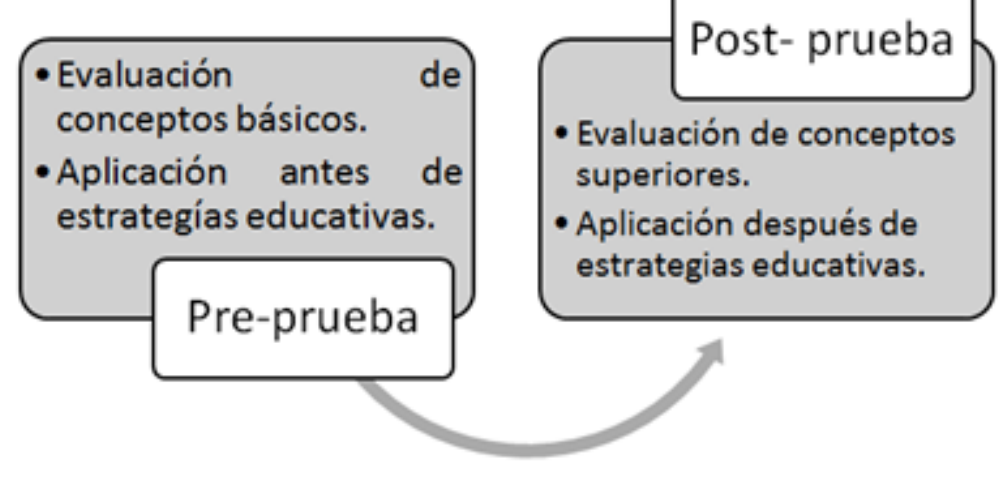

Figura 1: Características de la Pre-prueba y la Post- prueba. Fuente: elaboración propia.

Asimismo, una vez aplicadas la pre-prueba y la post-prueba, se lleva a cabo la valoración de la variable de investigación basada en la dimensión, subdimensión y en los indicadores que se muestran en la Tabla 1.

Tabla 1: Indicadores evaluados en la pre-prueba y la post-prueba. Fuente: elaboración propia.

\begin{tabular}{|c|c|c|c|}
\hline Variable & Dimensión & Subdimensión & Indicadores \\
\hline $\begin{array}{l}\text { Aprendizaje } \\
\text { de las } \\
\text { matemáticas. }\end{array}$ & $\begin{array}{l}\text { Aprendizaje de los } \\
\text { polígonos en el } \\
\text { plano cartesiano. }\end{array}$ & $\begin{array}{l}\text { - Polígonos } \\
\text { - Plano cartesiano } \\
\text { - Transformaciones en } \\
\text { el plano cartesiano: } \\
\text { traslación, rotación y } \\
\text { simetría central. }\end{array}$ & $\begin{array}{l}\text { - Ubica puntos en el plano } \\
\text { cartesiano. } \\
\text { - Representa polígonos en } \\
\text { el plano cartesiano. } \\
\text { - Realiza movimientos de } \\
\text { traslación, rotación y simetría } \\
\text { central de polígonos en el plano. }\end{array}$ \\
\hline
\end{tabular}

Teniendo en cuenta lo anterior, la valoración de la pre- prueba y la post- prueba, se realiza considerando un puntaje de uno (1) a cinco (5), distribuyéndose de la manera descrita en la Tabla 2.

Tabla 2: Valoración de la pre-prueba y la post-prueba. Fuente: elaboración propia.

\begin{tabular}{cccc}
\hline \multicolumn{2}{c}{ Pre-prueba } & \multicolumn{2}{c}{ Post-prueba } \\
\hline Ítem & Puntuación & Ítem & Puntuación \\
1 & 0,5 & 1 & 0,5 \\
2 & 2 & 2 & 1,5 \\
3 & 0,25 & 3 & 1 \\
4 & 1 & 4 & 1 \\
5 & 1,25 & 5 & 1 \\
Total & 5 & Total & 5 \\
\hline
\end{tabular}

El análisis estadístico de esta investigación respecto a la pre- prueba y post-prueba, se realiza encontrando los promedios y la desviación estándar. A su vez, para mayor confiabilidad, se usa la distribución normal para evaluar los efectos producidos por los recursos tecnológicos en el aprendizaje de las matemáticas, específicamente en el tema de polígonos en el plano cartesiano. 
Por último, la población tomada para realizar esta investigación estuvo conformada por 101 estudiantes de séptimo $\left(7^{\circ}\right)$ grado de la Educación Básica y que se encuentran matriculados en el Colegio Distrital San Vicente de Paúl. A continuación, en la Tabla 3, se detalla la distribución de los educandos.

Tabla 3: Distribución de la Población. Fuente: elaboración propia.

\begin{tabular}{cccc}
\hline Grupo & Femeninas & Masculinos & Total de alumnos \\
\hline $7^{\circ} \mathrm{A}$ & 15 & 19 & 34 \\
$7^{\circ} \mathrm{B}$ & 13 & 21 & 34 \\
$7^{\circ} \mathrm{C}$ & 17 & 16 & 33 \\
Total & 45 & 56 & 101 \\
\hline
\end{tabular}

Con base en esto, la muestra para la investigación quedó constituida por 64 estudiantes, los cuales pertenecen a dos grupos, 32 estudiantes en el grupo control $\left(7^{\circ} \mathrm{B}\right)$ y 32 alumnos en el grupo experimental $\left(7^{\circ} \mathrm{C}\right)$. Se aclara que dos $(2)$ estudiantes pertenecientes a $7^{\circ} \mathrm{B}$ y un (1) estudiante de $7^{\circ} \mathrm{C}$, se retiraron de la Institución y no participaron en el proceso de investigación. El grupo $7^{\circ} \mathrm{A}$ no fue tomado en cuenta debido a que la carga académica del área de matemáticas le pertenece a otra docente, razón por la cual, el tiempo y el espacio de trabajo no está acorde con los grupos escogidos. Se aclara de igual forma que los horarios de $7^{\circ} \mathrm{B}$ y $7^{\circ} \mathrm{C}$, presentan cruces con el tiempo de $7^{\circ} \mathrm{A}$.

\section{Discusión de los resultados}

$\mathrm{Al}$ aplicar el primer instrumento de investigación llamado "cuestionario sobre tecnologías de información y comunicación", se encontraron aspectos muy importantes que se resumirán con el fin de dar una visión general. Veamos algunos de ellos:

- El 60,9\% de los alumnos no utilizan la internet para aprender matemáticas. Además, 71.9\% de los estudiantes no recurren a la utilización de programas en la Web para aprender matemáticas, lo que significa que la mayor parte de los trabajos matemáticos son realizados de manera manual.

- El 75.0\% de alumnos no muestran interés por emplear hardware con la finalidad de almacenar información relevante de sus estudios y el 89.1 \% de estos hacen notar que nunca utilizan ellos mismos programas para estudiar temáticas en el aula de clases.

- El $100 \%$ de los estudiantes no han recibido nunca clases por medio de pizarras electrónicas o Pizarras Digitales Interactivas (PDI), por lo cual, el mismo porcentaje es obtenido al cuestionar a cerca de compartir sus conocimientos y usar a la vez dicho instrumento en alguna clase de la escuela.

- El cuestionario revela que el $85.9 \%$ de los alumnos nunca utilizan las Tecnologías de la Información y la Comunicación para aprender sin que el docente lo indique e incluso, el $53.1 \%$ de ellos, nunca busca en la Web contenidos por cuenta propia.

- Un $68.8 \%$ de los estudiantes nunca utiliza el computador para estudiar temas de matemáticas, incluso, los porcentajes respecto a los temas como usar programas computacionales para comprobar resultados matemáticos arrojó un $31.3 \%$ para casi nunca y un $43.8 \%$ para nunca en el cuestionario. 


\subsection{Resultados obtenidos al aplicar la Pre-prueba}

Para conocer el nivel de apropiación de los estudiantes respecto a los polígonos y los movimientos de estos en el plano cartesiano, se aplicó la pre-prueba en ambos grupos, control $\left(7^{\circ} \mathrm{B}\right)$ y Experimental $\left(7^{\circ} \mathrm{C}\right)$ conformado por 32 estudiantes cada uno. Se aclara nuevamente que la pre-prueba está conformada por preguntas con múltiples opciones de respuesta y preguntas abiertas, estas con el fin de observar los conceptos básicos que poseen los estudiantes y también, cómo realizan procedimientos de construcción geométrica utilizando instrumentos como regla, compás y transportador, de la misma forma, los alumnos tienen acceso a un computar con GeoGebra para realizar algunos ejercicios de forma digital. Los desempeños para la valoración de la pre-prueba corresponden a: básico (calificaciones de $1.0-2.9$ ), suficiente (calificaciones de $3.0-4.0$ ) y excelente (calificaciones de $4.1-5.0$ ), en la Figura 2 se detalla los porcentajes de alumnos que pertenecen a cada desempeño obtenido en la preprueba y también la distribución por grupo.

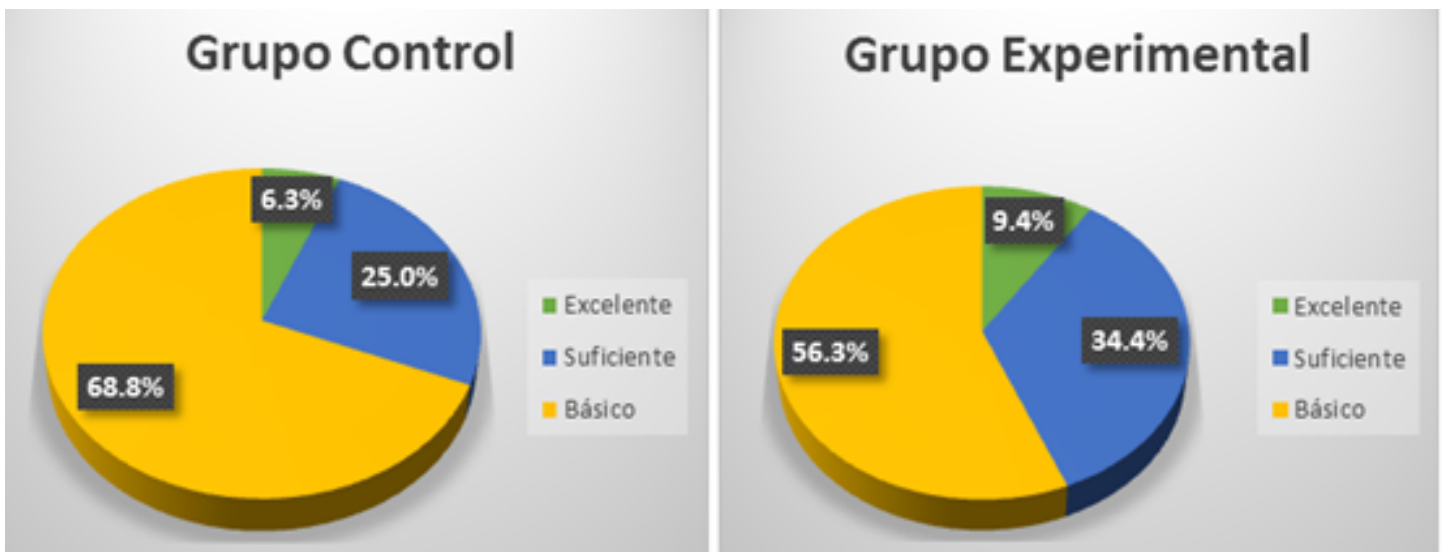

Figura 2: Porcentajes que representan el número de alumnos según el desempeño obtenido de la Pre-Prueba para cada grupo. Fuente: Elaboración propia.

Con base en los resultados obtenido al aplicar la Pre-prueba se observa que:

\section{Grupo Control}

- Dos (2) de los estudiantes demostraron tener los conocimientos y habilidades necesarias en la asignatura.

- Ocho (8) de los alumnos mostraron tener conocimientos suficientes para afrontar los conceptos previos respecto al tema evaluado.

- Veinte dos (22) educandos poseen habilidades mínimas para poner en práctica sus conocimientos respecto al tema.

\section{Grupo experimental}

- Tres (3) de los estudiantes lograron aplicar sus conocimientos y habilidades de manera satisfactoria.

- Once (11) de los alumnos poseen los conocimientos y habilidades suficientes para responder sobre el tema en cuestión.

- Dieciocho (18) educandos cumplen con los conocimientos básicos para responder acerca del tema. 
Entre los estudiantes que poseen los conocimientos mínimos, según puede observarse en la Figura 2, el grupo control arroja el $68.8 \%$ y el grupo experimental el $56.3 \%$; de la misma forma y respectivamente, aquellos que tienen los conocimientos suficientes están entre el $25 \%$ y $34.4 \%$, mientras los que cumplen con las capacidades cognitivas de manera satisfactoria están entre el $6.3 \%$ y $9.4 \%$, asimismo, cabe resaltar que usando una escala de 1 a 5 puntos, se obtuvo como promedio y desviación estándar para cada grupo: 3.38 y 0.609 para el grupo control; 3.53 y 0.671 para el grupo experimental, lo que significa un nivel de desempeño básico para ambos grupos.

\subsection{Resultados obtenidos al aplicar la Post-prueba}

Para obtener los resultados después de la aplicación de las estrategias de aprendizaje, se empleó la post-prueba. Cabe señalar que, en el desarrollo del curso, en el grupo experimental, se utilizó algunos softwares como: GeoGebra, Symbolab, la internet como medio para explorar blogs, video tutoriales, entre otros; mientras que el grupo control usó los procedimientos manuales para estudiar los polígonos en el plano cartesiano y los movimientos de translación, rotación y simetría central.

Haciendo un análisis general respecto a la post-prueba se obtuvo que el nivel excelente tuvo 22 alumnos (igual a $68.75 \%$ ) en el grupo experimental, contrario al grupo control que logró tener 9 alumnos en el mismo nivel (igual a $28.12 \%$ ). Entre los dos grupos, es el experimental quien registró el $6.25 \%$ (equivalente a 2 estudiantes) como resultado más bajo en comparación al grupo control con $31.25 \%$ (equivalente a 10 estudiantes). Aunque en el nivel suficiente, el grupo control tenga 13 alumnos (igual al $40.62 \%$ ) y el grupo experimental posea 8 alumnos (igual al $25 \%$ ), cabe resaltar que la mayor parte de los educandos se centra en el nivel excelente para el segundo grupo.

Con base en la información anterior y utilizando la misma escala en la post-prueba (de 1 a 5 puntos), el promedio y la desviación estándar para el grupo control es 3.97 y 0.782 y, para el grupo experimental es 4.63 y 0.609 , respectivamente, lo que significa que el primer grupo no logró por décimas llegar a un nivel suficiente sino mantenerse en un nivel básico, muy contrario al segundo grupo que logró ubicarse en un nivel de desempeño superior. Asimismo, se hace a continuación una comparación entre los resultados de la pre y post prueba, para observar el incremento o descenso porcentual que tuvieron ambos grupos en relación a los conocimientos y habilidades adquiridas durante la aplicación de estrategias de enseñanza y aprendizaje (ver Figura 3).

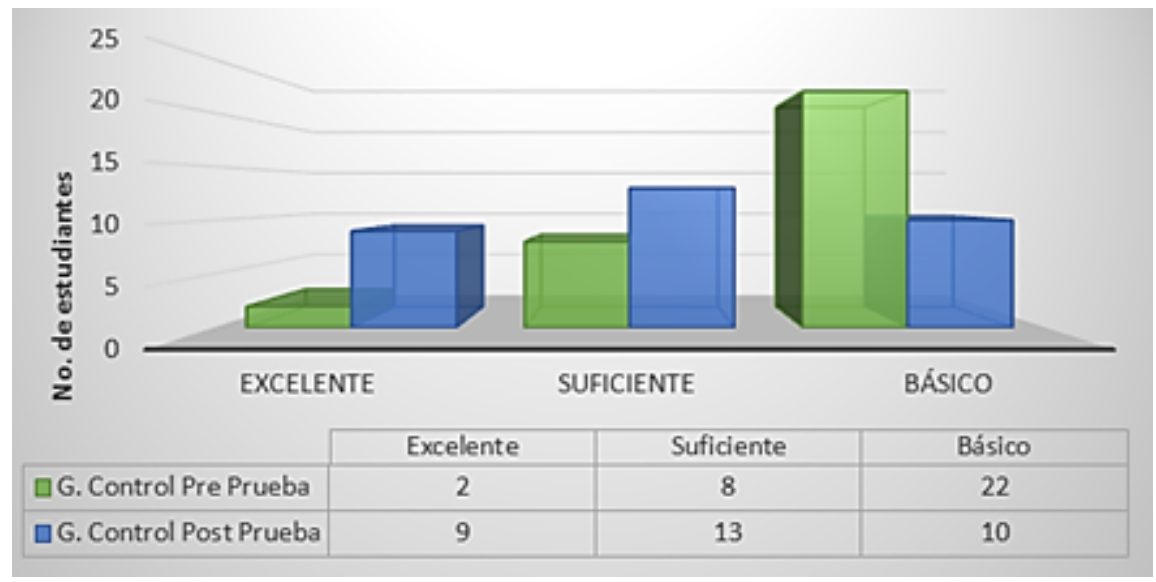

Figura 3: Comparación Pre y Post Prueba en el Grupo Control. Fuente: Elaboración propia.

En la Figura 3 se puede observar el aumento de estudiantes en los dos primeros niveles (Excelente y Suficiente), lo que representa para el primer nivel un incremento del $21.8 \%$ y para el segundo un $15.62 \%$, sin embargo, el tercer nivel (Básico) decrece en un $37.5 \%$, lo que demuestra un pequeño cambio en el aprendizaje dentro del área las matemáticas aplicando los procedimientos usuales para el estudio del concepto de polígonos y los movimientos de éstos en el plano cartesiano. 
Al discutir y analizar los resultados del grupo experimental (Figura 4), podemos notar que el nivel Excelente tuvo un incremento significativo del $59.37 \%$, muy por encima del porcentaje arrojado en el grupo control. El nivel Suficiente decreció en un 9.37\%, sin embargo, el descenso del nivel Básico fue del $50 \%$, porcentaje que es importante respecto al grupo control. Cabe resaltar que la gran concentración de los alumnos se encuentra en el primer nivel, lo que permite comprender el avance satisfactorio del grupo experimental gracias a la aplicación de las estrategias pedagógicas enfocadas en las Tecnologías de la Información y la Comunicación (ver Figura 4).

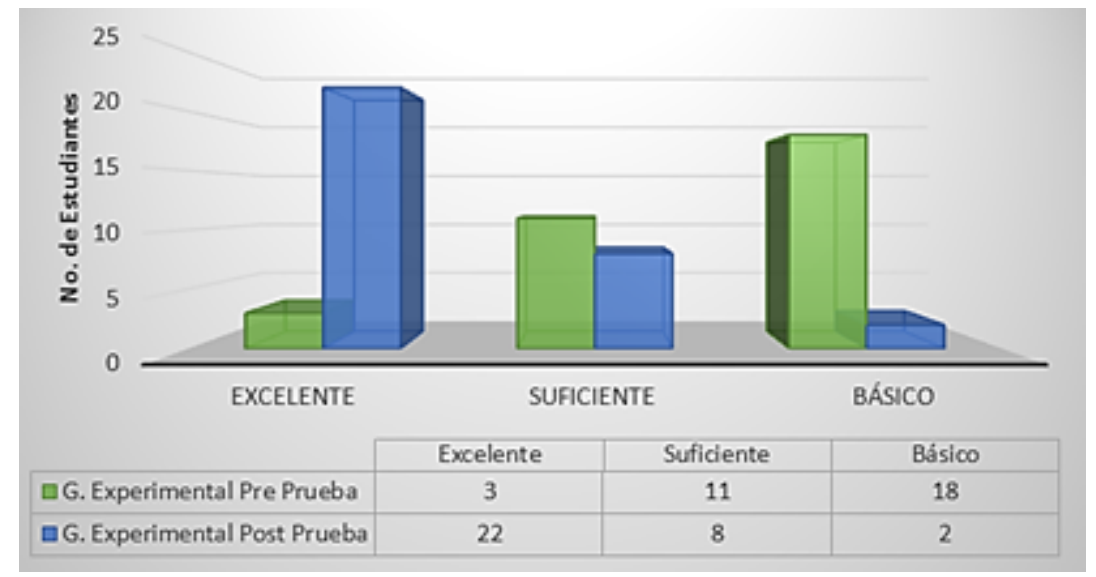

Figura 4: Comparación Pre y Post Prueba en el Grupo Experimental. Fuente: Elaboración propia.

Por consiguiente, se aplicó la distribución normal para hacer un comparativo sobre las probabilidades obtenidas en la pre y post prueba de cada grupo, control y experimental, teniendo en cuenta que ambos están conformados por 32 alumnos y que la calificación aprobatoria es de 3 a 5 puntos. Asimismo, se recopiló la información obtenida hasta el momento, la cual se presenta en la Tabla 4.

Tabla 4: Información general obtenida al aplicar la Pre y Post Prueba. Fuente: elaboración propia.

\begin{tabular}{lcccc}
\hline Grupos & \multicolumn{2}{c}{ Pre-prueba } & \multicolumn{2}{c}{ Post-prueba } \\
\hline & $\mu$ & $\sigma$ & $\mu$ & $\sigma$ \\
Grupo Control & 3.38 & 0.609 & 3.53 & 0.671 \\
Grupo Experimental & 3.97 & 0.782 & 4.63 & 0.609 \\
\hline
\end{tabular}

Si se aplica la distribución normal teniendo en cuenta el promedio $(\mu)$, la desviación estándar $(\sigma)$ y la nota aprobatoria mayor a 3 puntos para el grupo control con respecto a la pre y post-prueba, se tiene los siguientes valores:

- Pre-prueba; la probabilidad para $\mathrm{P}(\overline{\mathrm{x}}>3)=\mathrm{P}\left(\mathrm{Z}>\frac{\overline{\mathrm{x}}-\mu}{\sigma}\right)=\mathrm{P}\left(\mathrm{Z}>\frac{3-3.38}{0.609}\right)=$ $\mathrm{P}(\mathrm{Z}>-0.62)=\mathrm{P}(\mathrm{Z}<0.62)=0.7324 \approx 73.24 \%$

- Post-prueba; la probabilidad para $P(\bar{x}>3)=P\left(Z>\frac{\bar{x}-\mu}{\sigma}\right)=P\left(Z>\frac{3-3.53}{0.671}\right)=$ $\mathrm{P}(\mathrm{Z}>-0.78)=\mathrm{P}(\mathrm{Z}<0.78)=0.7823 \approx 78.23 \%$

Con los datos obtenidos hasta el momento se observa que el incremento porcentual entre la pre y post prueba para el grupo control fue $4.99 \%$. Al realizar el mismo análisis al grupo experimental, se tiene:

- Pre-prueba; la probabilidad para $P(\bar{x}>3)=P\left(Z>\frac{\bar{x}-\mu}{\sigma}\right)=P\left(Z>\frac{3-3.97}{0.782}\right)=$ $\mathrm{P}(\mathrm{Z}>-1.24)=\mathrm{P}(\mathrm{Z}<1.24)=0.8925 \approx 89.25 \%$ 
- Post-prueba; la probabilidad para $\mathrm{P}(\overline{\mathrm{x}}>3)=\mathrm{P}\left(\mathrm{Z}>\frac{\overline{\mathrm{x}}-\mu}{\sigma}\right)=\mathrm{P}\left(\mathrm{Z}>\frac{3-4.63}{0.609}\right)=$ $\mathrm{P}(\mathrm{Z}>-2.67)=\mathrm{P}(\mathrm{Z}<2.67)=0.9961 \approx 99.62 \%$

Se observa entonces que el incremento porcentual para el grupo experimental es de un $10.36 \%$, valor por encima del grupo control. Sin embargo, el porcentaje del post prueba en el grupo experimental es de $99.62 \%$, muy cercano al $100 \%$. Lo anterior significa que el grado de confiabilidad tiene una diferencia del $0.38 \%$ que, en comparación al grupo control, el resultado del post prueba tiene un porcentaje de $78.23 \%$ y la diferencia en cuanto a su confiabilidad es de $21.77 \%$. Razón que permite expresar como resultado final que la aplicación de los recursos tecnológicos en el aprendizaje de las matemáticas, específicamente en el estudio de los polígonos y los movimientos en el plano cartesiano, produce un aprendizaje satisfactorio.

\section{A modo de conclusión}

La aplicación de las estrategias pedagógicas usando recursos tecnológicos, mostró que el grupo experimental comprendía mejor los conceptos geométricos, identificando de manera eficiente características específicas del tema. Además, el grupo control, mostró dificultades para hacerlo dado que el trabajo realizado fue mecánico ya que debían utilizar herramientas como regla, compás y transportador, lo que fue rechazado de alguna manera por los alumnos debido a lo tedioso que resultaban los procesos de estudio.

Cabe resaltar que, gracias a la utilización de softwares con el grupo experimental, se logró observar algunos aspectos importantes, como: i) corregir errores de manera instantánea, ii) practicar constantemente sin esfuerzos manuales y iii) comparar los resultados obtenidos con otros compañeros de forma rápida. Después de realizar los procesos matemáticos (geométricos) paso a paso en el programa, el grupo experimental aprendió a realizar procedimientos de manera directa y eficaz, lo que reforzó mucho más sus conocimientos del tema, por el contrario, el grupo control, mostró dificultades para realizar sus procedimientos de manera manual al utilizar la regla, el compás y el transportador. Esto último resultaba ser complejo y molesto puesto que, para ellos, según su manifestación, requería un esfuerzo grande para no cometer errores, y si los había, no lograban encontrarlos.

Por otra parte, teniendo en cuenta que los alumnos debían obtener una calificación mayor que 3 puntos, se concluyen los siguientes aspectos:

\section{- Grupo Control.}

El porcentaje de los estudiantes con calificaciones superiores a 3 puntos en la pre-prueba era de un $73.24 \%$ que, luego de la aplicación de las estrategias usuales en el aula de clases aumentó en a $78.23 \%$ en la post-prueba. Lo que significa que el incremento porcentual es de $4.99 \%$.

\section{- Grupo Experimental.}

El porcentaje de los estudiantes con calificaciones superiores a 3 puntos en la pre-prueba era de un $89.25 \%$ que, al aplicar las estrategias pedagógicas usando las Tecnologías de la Información y la Comunicación ascendió a un $99.62 \%$ en la post-prueba. Lo que significa que el incremento porcentual es de $10.37 \%$.

Con base en la información anterior, se logra comprobar que los grupos que tienen acceso a las Tecnologías de la Información y Comunicación en el aula de clases, potencializan sus conocimientos y habilidades, mejorando no solo su desempeño sino su actitud de trabajo dentro y fuera de la escuela. Además, los efectos de las TICs en el aprendizaje de las matemáticas tienden a llevar a los alumnos a visualizar los contenidos de la asignatura desde otras perspectivas y a obtener resultados de aprendizaje satisfactorios. 


\section{Bibliografía}

[1] Aguiar, B., Velázquez, R. y Aguiar, J. (2019). Innovación docente y empleo de las TIC en la Educación Superior. Revista Espacios, 40 (2), pp. 8-19. https: //www. revistaespacios. com/a19v40n02/19400208.html.

[2] Aparicio, O. (2018). Las TIC como herramientas cognitivas. Revista Interamericana De Investigación, Educación., 11(1), pp. 67-80. https://doi.org/10.15332/s1657-107X. 2018. 0001.07.

[3] Cala, R., Díaz, L., Espí, N., y Tituaña, J. (2018). El impacto del uso de pizarras digitales interactivas (PDI) en el proceso de enseñanza aprendizaje. Un caso de estudio en la Universidad de Otavalo. Información Tecnológica, 29(5), pp. 61-70. http://dx. doi .org/10. 4067/S0718- 07642018000500061.

[4] Fernández, I., Riveros, V., y Montiel, G. (2017). Software educativo y las funciones matemáticas. Una estrategia de apropiación. Omnia, 23(1), pp. 9-19. https://www. redalyc. org/pdf/737/73753475002. pdf.

[5] García, L. (2019). Necesidad de una educación digital en un mundo digital. RIED. Revista Iberoamericana de Educación a Distancia, 22(2), pp. 09-22. doi: http://dx. doi .org/10. 5944/ried.22.2.23911.

[6] González, M., Abad, E., y Belmonte, L. (2020). Meaningful learning in the development of digital skills. Trend analysis. IJERI: International Journal of Educational Research and Innovation, (14), pp. 91-110. https://doi.org/10.46661/ijeri.4741.

[7] Gutiérrez, M. (2018). Estilos de aprendizaje, estrategias para enseñar. Su relación con el desarrollo emocional y . ${ }^{a}$ prender a aprender". Tendencias Pedagógicas, 31, pp. 83-96. http://dx.doi.org/10.15366/tp2018.31.004

[8] Hermann, A., Apolo, D. y Molano, M. (2019). Reflexiones y Perspectivas sobre los Usos de las Redes Sociales en Educación. Un Estudio de Caso en Quito-Ecuador. Revista Información Tecnológica, 30 (1), pp. 215-224.

[9] Hernández, R., y Mendoza, C. (2018). Metodología de la investigación: las rutas cuantitativa, cualitativa y mixta. Mc Graw-Hill Editores.

[10] Jama, V., y Cornejo, J. (2016). Los recursos tecnológicos y su influencia en el desempeño de los docentes. Dominio de las Ciencias, 2, pp. 201-219. https://dialnet. unirioja.es/ servlet/articulo? codigo $=6324010$.

[11] Jiménez, J., y Jiménez, S. (2017). GeoGebra, una propuesta para innovar el proceso enseñanza-aprendizaje en matemáticas. Revista Electrónica Sobre Tecnología, Educación Y Sociedad, 4(7). Recuperado a partir de https://www.ctes.org.mx/index.php/ctes/ article/view/654

[12] Ley N 115. Congreso de la República de Colombia, Santa Fe de Bogotá, Colombia. 8 de febrero de 1994. http://www. mineducacion.gov.co/1621/articles-85906_archivo_pdf. pdf

[13] Ministerio De Educación Nacional. (2002). Estándares Básicos de Competencias en Leguaje, Matemáticas, Ciencias y Ciudadanas. Guía sobre lo que los estudiantes deben saber y saber hacer con lo que aprenden. Revolución Educativa Colombia Aprende. Primera edición. 
[14] Rodríguez, J., Romero, J., y Vergara, G. (2017). La importancia de las TIC en la enseñanza de las matemáticas. Matua Revista del Programa de Matemáticas, 4(2), pp. 19. http://investigaciones. uniatlantico.edu.co/revistas/index.php/MATUA/article/ view/1861.

[15] Suárez, Y. (2018). Consolidación de una línea de investigación en TIC y educación matemática. TIC y políticas públicas en educación. Su incidencia en el aula de clase. Universidad de Carabobo, Dirección de Medios y Publicaciones.

[16] Vázquez, D. (2020). Nuevos paradigmas educativos, económicos y sociales para la sostenibilidad. Ecocience International Journal, 2(3), pp. 14-21. https://doi .org/10.35766/ je20232. 